\title{
Surgical Trauma and Postoperative Immune Dysfunction
}

\author{
P. Menges W. Kessler C. Kloecker M. Feuerherd S. Gaubert S. Diedrich \\ J. van der Linde A. Hegenbart A. Busemann T. Traeger K. Cziupka \\ C.-D. Heidecke S. Maier \\ Department of Surgery, University Hospital Greifswald, Greifswald, Germany
}

\section{Key Words}

Immune system • Postoperative immune suppression •

Trauma $\cdot$ Sepsis

\begin{abstract}
Background: In postoperative sepsis, mortality is increased due to the surgically induced immune dysfunction. Further causes of this traumatic effect on the immune system include burn injuries and polytrauma, as well as endogenous traumata like stroke. Several animal models have been defined to analyse the characteristics of trauma-induced immune suppression. This article will correlate our results from animal studies and clinical observations with the recent literature on postoperative immune suppression. Methods: The previously described model of surgically induced immune dysfunction (SID) was performed in mice by laparotomy and manipulation of the small intestine in the antegrade direction. Blood samples were collected 6 and $72 \mathrm{~h}$ following SID to analyse the white blood cell count and corticosterone levels. To assess the postoperative immune status in humans, we analysed expression of HLA-DR on monocytes of 118 patients by flow cytometry prior to and 24,48 and $72 \mathrm{~h}$ after surgery. Results: The postoperative immune suppression in our SID model is characterised by lymphocytopenia and significantly increased corticosterone levels in mice dependent on the degree of surgical trauma. This is comparable to the postoperative situation in humans: major and
\end{abstract}

especially long-lasting surgery results in a significantly reduced expression of HLA-DR on circulating monocytes. Previous studies describe a similar situation following burn injury and endogenous trauma, i.e. stroke. Conclusions: We suggest the completion of our previously published sepsis classification due to the immune status at the onset of sepsis: type $A$ as the spontaneously acquired sepsis and type $B$ as sepsis in trauma-induced pre-existing immune suppression.

Copyright $\odot 2012$ S. Karger AG, Basel

\section{Introduction}

The efficient function of the immune system is essential for an organism that is confronted with pathogens. The innate immunity represents the first line of host defence and includes macrophages, monocytes, neutrophils and dendritic cells. They perform phagocytosis as well as cytokine release and therefore activate the adaptive immune system. This adaptive or specific immune system provides the ability to generate immunity by lymphocytic antigen production [1].

The innate immune system is not only activated by pathogen-associated molecular patterns, but also by surgical stress and trauma, i.e. chest trauma, fracture or

P.M. and W.K. contributed equally to this work.

\section{KARGER \\ Fax +4161306 1234 E-Mail karger@karger.ch} www.karger.com

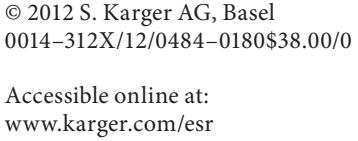

Pia Menges, MD

Department of Surgery: General Surgery, Visceral, Thoracic and Vascular Surgery

University Hospital Greifswald

Sauerbruchstrasse, DE-17475 Greifswald (Germany)

Tel. +49383486 6001, E-Mail pia.menges@uni-greifswald.de 
brain injury $[2,3]$. In this case, so-called danger-associated molecular patterns have been identified as danger signals that mediate the early post-traumatic inflammatory response [4]. Both pathogen- and danger-associated molecular patterns are recognised by immunologically competent cells that express receptors on their surface, such as Toll-like receptors [5].

This inflammatory reaction based on a nonpathogenic activation results in a temporary post-traumatic or postoperative immune suppression which predisposes for septic complications [6]. The postoperative immune suppression is associated with a reduction in the expression of HLA-DR on monocytes. This is accompanied by an impaired ability of monocytes to respond to challenge with LPS and with a reduced proliferation by stimulated Tlymphocytes [7-9]. Irrespective of the nature of the surgical procedure used, all patients suffer from postoperative dysfunction of monocytes [10]. Several studies have compared laparoscopic and conventional surgery and described a direct correlation between the extent of trauma and the degree of postoperative immune suppression $[11,12]$.

The postoperative immune dysfunction is already measurable on the first day after surgery. In the case of an uncomplicated postoperative course, the normal immune function is re-established within a few days depending on the extent and severity of the surgical procedure. In postoperative sepsis there is a direct correlation between suppression of monocyte HLA-DR expression and severity of the septic syndrome which is defined as the presence of both a systemic inflammatory response and infection $[10,13,14]$. The mortality of septic patients in European intensive care units still ranges from 10 to $35 \%$ despite all recent medical progress [15]. Therefore, sepsis remains a severe problem in postoperative patient management [16]. It is important to further investigate the origins of sepsis and, in particular, the contribution of the immune status of the patient to sepsis onset.

Mortality in sepsis is significantly lower if sepsis is acquired spontaneously (i.e. perforated diverticulitis) compared to postoperative septic complications like pneumonia or anastomosis insufficiency $[13,17,18]$. Therefore, sepsis is classified referring to the immune function status at the moment of its beginning: spontaneously acquired sepsis (type A) develops in the presence of an intact immune system, whereas postoperative sepsis (type B) develops in the context of a partially disabled immune system [19].

Several animal models have been characterised to investigate the mechanisms of postoperative and post-traumatic immune suppression. Our previously described model of surgically induced immune dysfunction (SID) is based on the intestinal trauma of an abdominal surgery procedure [20]. The intestine of mice is pressed smoothly, three times consecutively to induce a significant immune suppression as detected by the impaired ex vivo cytokine release of splenocytes [21]. Similar observations of trauma-induced immune suppression were made in mice with burn wounds: the concentration of peripheral blood leucocytes declined several hours following the trauma [22]. In addition, haemorrhage trauma influences the immune function and this effect was shown to involve a Toll-like receptor 4 pathway in mice $[23,24]$. Even apoplectic stroke seems to influence the immune status as pneumonia occurring subsequent to stroke raises the mortality rate [25].

In this review we correlate the published results from animal studies with clinical observations on postoperative immune suppression. We discuss mechanisms and strategies to prevent postoperative immune dysfunction. Finally, we suggest a new arrangement of the sepsis classification based on the immune status at development.

\section{Methods}

\section{Animal Model of SID}

All animal experiments were designed and carried out according to the guidelines of the German Animal Protection Act. Permission was obtained from the governmental committee on animal welfare (LALLF M-VL/TSD/7221.3-1.1-037/07). As described before [21], we laparotomised female C57BL/6 mice aged $8-12$ weeks with an average body weight of $22 \mathrm{~g}$. The small intestine was pressed smoothly between two sterile Q-tips, three times consecutively. In the laparotomy group, the abdomen was laid open and closed after several minutes without touching the intestine.

\section{Analysis of Corticosterone Levels in Serum}

Following surgical trauma mice were anesthetised and blood was harvested by retro-orbital puncture $6 \mathrm{~h}$ after surgery. The samples were analysed by Corticosterone ELISA (Hoelzel Diagnostica, Cologne, Germany) according to the manufacturer's protocol ( $\mathrm{n}=5$ per group).

Detection of White Blood Cell Count in Mice

Blood was drawn by retro-orbital punction on EDTA tubes $72 \mathrm{~h}$ following SID or laparotomy in the control group. $50 \mu \mathrm{l}$ were analysed on an automatic cell counter for veterinary use (Vetscan; Scil Animal Care Company GmbH, Viernheim, Germany) calibrated for mice blood measurement ( $n=8$ per group).

Patient Population and Study Design

The procedures were conducted from April 2003 until April 2004 at the Department of Surgery, University Hospital Greifswald, Germany. For prospective data acquisition, blood was col- 
lected from 118 patients preoperatively and at 24,48 and $72 \mathrm{~h}$ following surgical procedure. Patients were aged 18 years or more and had a maximum ASA score of 4 . The criteria for exclusion were palliative surgery and diseases of acute inflammation. The study was conducted in accordance with the ethical standards of the Helsinki Declaration of 1975 and was approved by the university's ethics commission. Informed consent was obtained from all patients.

\section{Surgical Procedures: Major and Minor Surgery}

Seventeen patients (11 males and 6 females, aged $60 \pm 15$ years) formed the 'minor surgery' group, whereby 'minor surgery' was defined as resection of the gallbladder, thyroid gland or hernia repair. The mean operation duration was $1.58 \pm 0.47 \mathrm{~h}$ and the period of hospitalisation was $6.25 \pm 1.92$ days. One hundred and one patients (63 males and 38 females, aged $63.67 \pm 12.76$ years) were included in the 'major surgery' group and comprised thoracic surgery (pneumonectomy, lobectomy and segment resection), colorectal surgery (hemicolectomy, sigma resection, anterior rectum resection, abdominoperineal resection), oesophagus and gastric surgery, segment resection of the liver and pancreatic surgery (Whipple resection, Traverso resection, Beger resection). Eighty percent of the patients in this group underwent surgical therapy because of malignant disease. All patients underwent elective surgery and emergency operations were not included. For details see table 1.

\section{Isolation of Human Peripheral Blood Mononuclear Cells}

Human peripheral blood mononuclear cells (PBMCs) were isolated from $10 \mathrm{ml}$ of heparinised EDTA blood samples by Ficollmetrizoate density gradient centrifugation. Isolated cells were washed twice with PBS and the total cell count was determined. Cells were resuspended in PBS supplemented with $1 \%(\mathrm{v} / \mathrm{v})$ calf serum and $0.05 \% \mathrm{NaN}_{3}$, and CD14 microbeads (Miltenyi Biotech, Bergisch Gladbach, Germany) were added according to the manufacturer's instructions. Incubation at $4^{\circ} \mathrm{C}$ for $5 \mathrm{~min}$ was followed by magnetic separation using MiniMACS separation columns (Miltenyi Biotech).

\section{FACS Analysis of HLA-DR Expression on PBMCs}

Expression of HLA-DR on monocytes was analysed by threecolour immunofluorescence staining. Ten millimeters of heparinised blood were incubated with monoclonal CD14 antibody.

Erythrocytes were lysed by FACS Lysing Solution (Becton Dickenson, Heidelberg, Germany) and fluorescence was analysed on a FACS Calibur System (Becton Dickson). Instrument calibration was performed with Fluorosphere beads (DAKO Cytomation, Hamburg, Germany) according to the manufacturer's recommendations. PBMCs were identified by forward and side scatter profile and positive staining with antibodies to CD14.

\section{Statistical Analyses}

Data was analysed using Graph Pad Prism Version 3.02 for Windows (Graph Pad Software, San Diego, Calif., USA). Differences between two samples at one time point were analysed by unpaired t test as the data had a Gaussian distribution. We used $t$ test for paired samples to compare differences between consecutive blood samples. All data are expressed as mean \pm SEM; $\mathrm{p} \leq$ 0.05 was considered to be statistically significant.
Table 1. Classification of sepsis due to the status of immune function [18]

\begin{tabular}{lll}
\hline & $\begin{array}{l}\text { Type A, } \\
\text { spontaneously } \\
\text { acquired sepsis }\end{array}$ & $\begin{array}{l}\text { Type B, } \\
\text { post-traumatically } \\
\text { acquired sepsis }\end{array}$ \\
\hline Previous trauma & none & $\begin{array}{l}\text { surgical procedure, } \\
\text { stroke, accident, poly- } \\
\text { trauma, burn injury, } \\
\text { haemorrhagic shock }\end{array}$ \\
\hline $\begin{array}{l}\text { Failure of eradication } \\
\text { of the septic focus }\end{array}$ & unusual & usual \\
\hline Mortality & low & high \\
\hline Immune paralysis & no & yes \\
\hline Immune response & physiologic & suppressed \\
\hline
\end{tabular}

\section{Results}

We developed a murine model that reproduces the surgically induced immune dysfunction and in which the small intestine is pressed smoothly between two q-tips, three times consecutively. The influence of this surgical procedure on the immune status is described by the analysis of cytokine levels in serum and ex vivo cytokine release of splenocytes [21]. The immune status is additionally characterised by a significantly decreased white blood cell count 3 days following surgery when compared to mice that were laparotomised without touching the intestine. This effect was shown for leucocytes (fig. 1a, $p<$ $0.01, \mathrm{n}=8$ per group) and seems to originate in the reduced number of lymphocytes (fig. $1 \mathrm{~b}, \mathrm{p}<0.0001, \mathrm{n}=8$ per group). The levels of monocytes and neutrophils were not influenced following SID (data not shown).

Corticosterone in serum was analysed to describe another factor of the immune status. Six hours following SID we detected significantly increased corticosterone levels in serum when compared to the laparotomised control group (fig. $1 \mathrm{c}, \mathrm{p}<0.05, \mathrm{n}=5$ per group).

To define the postoperative immune status in human patients we analysed the expression of HLA-DR on PBMCs by flow cytometry. Preoperatively, there were no differences between major and minor surgery but, in accordance with a previously published report, we observed a significant difference between preoperative and postoperative HLA-DR expression levels [26].

Surgery by itself leads to a highly significant ( $\mathrm{p}<$ 0.0001 ) reduction of HLA-DR expression in both groups. 


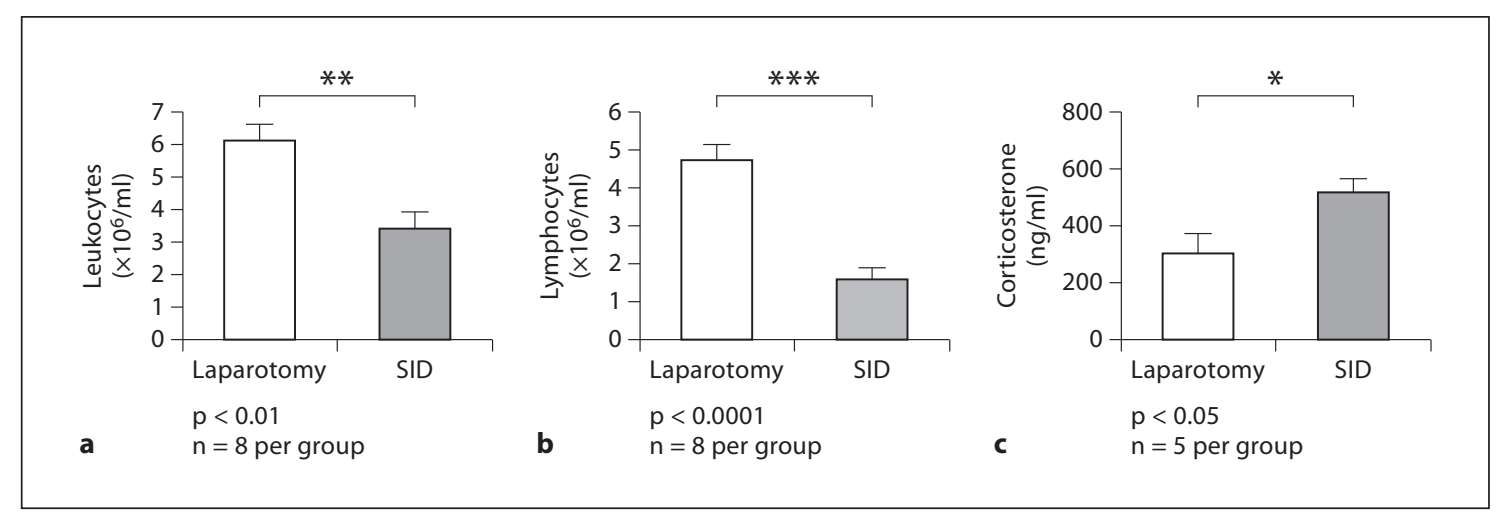

Fig. 1. Lymphocytopenia and elevated corticosterone levels confirm the status of postoperative immune suppression in the surgically induced immune dysfunction model. To describe the influence of surgical trauma on immune status we developed a murine model that reproduces the SID: the small intestine is pressed smoothly between two Q-tips, three times consecutively. Mice were laparotomised in the control group without the intestine being touched. Blood samples were collected at different points in

Significant differences $(\mathrm{p}<0.0005)$ between the major and minor surgery groups were evident $24 \mathrm{~h}$ after surgery (fig. 2a). Over the course of the next $48 \mathrm{~h}$ we observed a slight increase of HLA-DR expression in both major and minor surgery patients though the difference between the groups remained nearly unchanged.

To analyse the influence of operation duration on postoperative HLA-DR expression of human monocytes, we compared 'long-term surgery' ( $>2.5 \mathrm{~h}$ in length) to 'short-term surgery' ( $<2.5 \mathrm{~h}$ in length) regardless of whether the surgery was major or minor (fig. $2 b$ ). In both groups HLA-DR levels on monocytes dropped compared to the preoperative level. Long-term surgery caused a significantly higher decline compared to that seen after shorter operations. This difference was highly significant $(\mathrm{p}<0.0005) 24 \mathrm{~h}$ after surgery.

\section{Discussion}

The mechanisms of the postoperative and post-traumatic immune dysfunction are still subject of numerous studies. Currently it is assumed that the upregulated inflammatory reaction to the trauma induces an inhibition of both macrophages and cell-mediated immune function which finally results in an increased predisposition of sepsis development after major surgery [27].

The activation of the hypothalamic-pituitary-adrenal axis and subsequent release of glucocorticoids such as time following the surgical procedure and the levels of white blood cells as well as corticosterone were detected. We observed a significantly decreased white blood cell count 3 days following surgery, as it is shown for leucocytes (a) and lymphocytes (b). Murine serum levels of corticosterone were significantly increased following SID compared to the control group when analysed $6 \mathrm{~h}$ postoperatively (c). ${ }^{*} \mathrm{p}<0.05 ;{ }^{* *} \mathrm{p}<0.01 ;{ }^{* *} \mathrm{p}<0.0001$.

cortisol (proportional to the severity of the surgical stress) is an important factor in the development of postoperative immune dysfunction. Glucocorticoids reduce the rate of T cell proliferation, induce lymphocytopenia and increase the expression of anti-inflammatory gene products [28]. This goes in line with our results from the SID model which confirm the increase of corticosterone levels.

The immunosuppressive effect of stress has been investigated extensively. Activation of the sympathetic nervous system results in the release of catecholamines which influence immune cells via adrenergic binding sites on their surface. Among others, T cell proliferation and NK cell cytotoxicity is inhibited by the activation of $\beta 2$-receptors $[29,30]$. Several studies describe that alterations in immune markers can be observed even before surgery [31]. This underlines the relevance of prevention of preoperative stress situations by arrangements such as short hospitalization to reduce psychological as well as physical stress.

We observed significant lymphocytopenia in the postoperative period in our model of SID. This correlates with the situation in humans following trauma where numerous studies characterise the immune suppression after traumatic injury [27, 32]. The numbers of white blood cells in the circulating blood increases while circulating lymphocyte numbers are reduced. The extent and duration of this phenomenon is related to the magnitude of the previous surgical procedure [33]. The postoperative impairment of $\mathrm{T}$ cell function with a shift to regulatory 
Fig. 2. HLA-DR expression on monocytes is significantly decreased following major or long-term surgery compared to minor/ short-term surgery. a Surgical procedures alone lead to significant $(\mathrm{p}<0.001)$ reduction of HLA-DR expression following minor and major surgery procedures. $24 \mathrm{~h}$ after surgery there are significant differences between both groups $(p<0.005)$. The level of HLA-DR expression shows a slight increase until $72 \mathrm{~h}$ after surgery, while the difference between both groups remained nearly unchanged. b To analyse the influence of operation duration on postoperative HLA-DR expression, we compared long-term surgery ( $>2.5 \mathrm{~h}$ in length) to short-term surgery ( $<2.5 \mathrm{~h}$ in length) regardless of whether the surgery was major or minor. In both groups HLA-DR levels on monocytes dropped significantly compared to the preoperative level $(\mathrm{p}<0.0005)$. Surgery that required more than $2.5 \mathrm{~h}$ led to a significantly higher decline compared to shorter operations. This difference was highly significant $(\mathrm{p}<0.0005) 24 \mathrm{~h}$ after surgery and was reduced 48 and 72 h postoperatively.

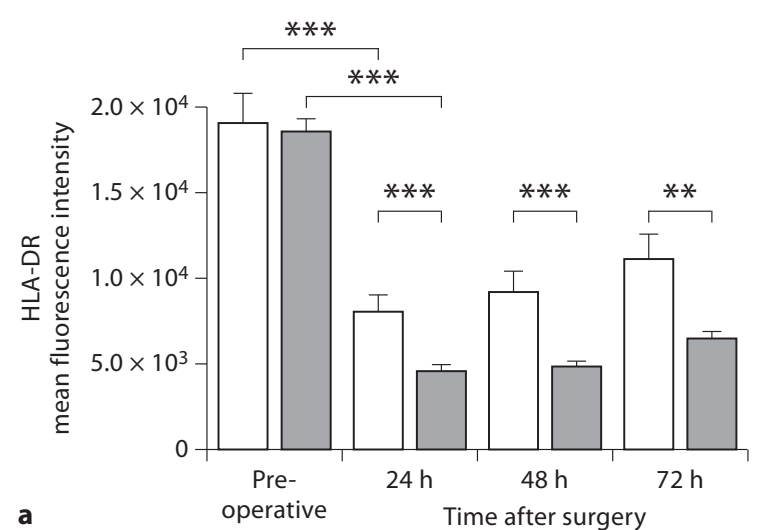

Minor surgery procedure $\mathrm{n}=17$

$\square$ Major surgery procedure $\mathrm{n}=101$

**p $<0.005$

*** $p<0.001$

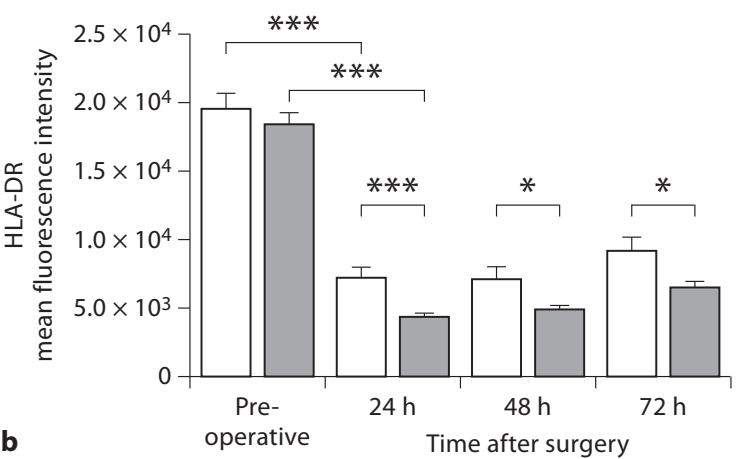

Short-term surgery $\mathrm{n}=30$

$\square$ Long-term surgery $\mathrm{n}=84$ $\begin{aligned}{ }^{*} p & <0.05 \\ * * * & <0.0005\end{aligned}$
T lymphocytes as well as a suppressed NK cell cytotoxicity supports the immune suppressive effect [34]. Moreover, the phagocytic function and motility of neutrophils is reduced and macrophage as well as monocyte function is inhibited [32]. This functional loss could be associated with the reduced HLA-DR expression on these cells.

Using surface expression of monocyte HLA-DR as a measure of immune suppression we could show that patients who underwent major surgery procedures had significantly more extensively suppressed monocyte function as compared to minor surgery patients. Both patient groups underwent similar regimes of anaesthesia and narcotic treatment but differed in the extent of tissue trauma, operation duration and blood loss. Twenty-four hours after surgery, patients with minor surgery had recovered significantly more surface expression of monocyte HLA-DR as compared to major surgery patients and, by this criterion, were less immune suppressed. These differences were evident even on postoperative day 3 .

This confirms previous studies that describe a direct correlation between the extent of trauma and the degree of postoperative immune suppression. Decreased im- mune function by extended surgical trauma has been shown by comparing laparoscopic and conventional surgery $[11,12]$. Following conventional surgery there is significantly less HLA-DR expression on monocytes as compared to patients undergoing laparoscopic surgery, indicating a relatively higher degree of immune suppression following conventional surgery [35]. Similarly, in colorectal surgery extended surgical trauma is followed by increased postoperative immune suppression, which is in accordance with our results [36].

The duration of the operation is another factor which has a significant influence on the postoperative immune status. Irrespective of the degree of surgical trauma involved, an operation lasting longer than $2.5 \mathrm{~h}$ resulted in a significantly lower monocyte HLA-DR expression than shorter operations.

The analysis of HLA-DR expression on monocytes seems to be a useful tool to quantify the immune status of patients. There is a negative correlation between surface expression of HLA-DR on monocytes and the incidence of infectious diseases in trauma patients [37], and a decreased HLA-DR expression in septic shock is associated with in- 
creased mortality risk $[38,39]$. In line with these studies we detected a direct correlation between postoperatively reduced HLA-DR expression and incidence of severe sepsis in the postoperative period (data not shown). Major, and especially protracted, surgery results in a significantly reduced expression of HLA-DR on circulating monocytes.

A patient's functional immune status will have a decisive role to play in the induction of postoperative septic complications. Prolonged disarming of the innate immune system is associated with a significantly increased incidence of septic complications [37, 38, 40]. Therefore, strategies to prevent postoperative immune suppression have to be developed and integrated into management, especially optimizing surgical techniques to realise a short duration of the surgical procedure. Minimally invasive surgery should be performed whenever possible and preoperative stress for the patient should be minimised. Adequate analgesic pain control should be taken for granted. There are also data that provide evidence for a positive effect of epidural anaesthesia on the postoperative immune status [41].

Previous studies describe stroke as another inductor of peripheral immune suppression which predisposes for subsequent bacterial infections that impair clinical outcome [42-44]. If pneumonia occurs subsequent to stroke, the mortality rate is increased [45].

The immunological changes following stroke are comparable to those in the postoperative period: they include inhibition of macrophage activity, lymphocytopenia and increased serum IL-10 concentrations. Several studies describe increased catecholamine and cortisol levels induced by stroke that correlate with the development of post-stroke infection [46]. We therefore conclude that the development of sepsis following surgery- and traumainduced immune suppression is comparable to septic complications subsequent to stroke. This understanding should lead to a rearrangement of the previously described sepsis classification.

The classification of abdominal sepsis is guided by the status of immune function at the development of sepsis: spontaneously acquired sepsis (type A) develops in the presence of an intact immune system, whereas postoperative sepsis (type B) develops in the context of a partially disabled immune system [19]. As there are impressive data on the effect of various traumata on the immune system we suggest extending the classification of sepsis type B to include sepsis that develops following any trauma- or injury-induced immune suppression, recognising polytrauma, burn injury, fracture and endogenous trauma like stroke. Type B sepsis should then be renamed as post-traumatically acquired sepsis (table 1 ). Type B sepsis is characterised by a suppressed immune response due to immune paralysis, commonly occurring failure to eradicate the septic focus and therefore a high mortality rate.

In conclusion, trauma-induced immune dysfunction is possibly based on the inflammatory activation of the immune function and stress is a significant cofactor. The trauma originates in surgical procedures, traumatic injuries and endogenous traumata like stroke. Sepsis that develops in the period of post-traumatic immune dysfunction should be classified as type B sepsis and is characterised by high mortality rates. Therefore, further studies are necessary to determine strategies that will help to minimize the incidence of this severe disease pattern.

\section{Acknowledgements}

We thank Antje Janetzko and Doreen Biedenweg for their excellent technical assistance. C.K., M.F. and A.H. were supported by a predoctoral fellowship in the graduate school GRK 840 (Host-pathogen interactions) by Deutsche Forschungsgemeinschaft (DFG).

\section{References}

1 Beutler B: Innate immunity: an overview. Mol Immunol 2004;40:845-859.

-2 Stahel PF, Smith WR, Moore EE: Role of biological modifiers regulating the immune response after trauma. Injury 2007;38:14091422.

-3 Neher MD, Weckbach S, Flierl MA, HuberLang MS, Stahel PF: Molecular mechanisms of inflammation and tissue injury after major trauma - is complement the 'bad guy'? J Biomed Sci 2011;18:90.
4 Matzinger P: The danger model: a renewed sense of self. Science 2002;296:301-305.

5 Akira S, Takeda K, Kaisho T: Toll-like receptors: critical proteins linking innate and acquired immunity. Nat Immunol 2001;2:675680.

-6 Cheron A, Floccard B, Allaouchiche B, Guignant C, Poitevin F, Malcus C, et al: Lack of recovery in monocyte human leukocyte antigen-DR expression is independently associated with the development of sepsis after major trauma. Crit Care 2010; 14:R208.
7 Faist E, Kupper TS, Baker CC, Chaudry IH, Dwyer J, Baue AE: Depression of cellular immunity after major injury: its association with posttraumatic complications and its reversal with immunomodulation. Arch Surg 1986;121:1000-1005.

-8 Faist E, Schinkel C, Zimmer S, Kremer JP, Von Donnersmarck GH, Schildberg FW: Inadequate interleukin-2 synthesis and interleukin-2 messenger expression following thermal and mechanical trauma in humans is caused by defective transmembrane signalling. J Trauma 1993;34:846-853. 
-9 Horgan AF, Mendez MV, O’Riordain DS, Holzheimer RG, Mannick JA, Rodrick ML: Altered gene transcription after burn injury results in depressed T-lymphocyte activation. Ann Surg 1994;220:342-351.

-10 Heidecke CD, Weighardt H, Hensler T, Bartels H, Holzmann B: Immune paralysis of Tlymphocytes and monocytes in postoperative abdominal sepsis. Correlation of immune function with survival (in German). Chirurg 2000;71:159-165.

- 11 Milsom JW, Bohm B, Hammerhofer KA, Fazio V, Steiger E, Elson P: A prospective, randomized trial comparing laparoscopic versus conventional techniques in colorectal cancer surgery: a preliminary report. J Am Coll Surg 1998;187:46-54.

-12 Stage JG, Schulze S, Moller P, Overgaard H, Andersen M, Rebsdorf-Pedersen VB, et al: Prospective randomized study of laparoscopic versus open colonic resection for adenocarcinoma. Br J Surg 1997;84:391-396.

-13 Hensler T, Hecker H, Heeg K, Heidecke CD, Bartels H, Barthlen W, et al: Distinct mechanisms of immunosuppression as a consequence of major surgery. Infect Immun 1997; 65:2283-2291.

-14 Levy MM, Fink MP, Marshall JC, Abraham E, Angus D, Cook D, et al: 2001 SCCM/ ESICM/ACCP/ATS/SIS International Sepsis Definitions Conference. Crit Care Med 2003;31:1250-1256.

15 Vincent JL, Sakr Y, Sprung CL, Ranieri VM, Reinhart K, Gerlach H, et al: Sepsis in European intensive care units: results of the SOAP study. Crit Care Med 2006;34:344353.

16 Brunkhorst FM: Epidemiology, economy and practice - results of the German study on prevalence by the competence network sepsis (SepNet). Anasthesiol Intensivmed Notfallmed Schmerzther 2006;41:43-44.

$\checkmark 17$ Chandra V, Nelson H, Larson DR, Harrington JR: Impact of primary resection on the outcome of patients with perforated diverticulitis. Arch Surg 2004;139:1221-1224.

- 18 Branagan G, Finnis D: Prognosis after anastomotic leakage in colorectal surgery. Dis Colon Rectum 2005;48:1021-1026.

-19 Maier S, Traeger T, Westerholt A, Heidecke CD: Special aspects of abdominal sepsis (in German). Chirurg 2005;76:829-836

$>20$ Kalff JC, Schraut WH, Simmons RL, Bauer AJ: Surgical manipulation of the gut elicits an intestinal muscularis inflammatory response resulting in postsurgical ileus. Ann Surg 1998;228:652-663.

-21 Koerner P, Busemann A, Traeger T, Kessler W, Cziupka K, Diedrich S, et al: Postoperative immune suppression in visceral surgery: characterisation of an intestinal mouse model. Eur Surg Res 2011;47:260-266.
22 Calum H, Moser C, Jensen PO, Christophersen L, Maling DS, van Gennip M, et al: Thermal injury induces impaired function in polymorphonuclear neutrophil granulocytes and reduced control of burn wound infection. Clin Exp Immunol 2009;156:102110.

-23 Reino DC, Pisarenko V, Palange D, Doucet D, Bonitz RP, Lu Q, et al: Trauma hemorrhagic shock-induced lung injury involves a gut-lymph-induced TLR4 pathway in mice. PLoS One 2011;6:e14829.

24 McGhan LJ, Jaroszewski DE: The role of tolllike receptor- 4 in the development of multiorgan failure following traumatic haemorrhagic shock and resuscitation. Injury 2012; 43:129-136

25 Vogelgesang A, Dressel A: Immunological consequences of ischemic stroke: immunosuppression and autoimmunity. J Neuroimmunol 2011;231:105-110.

26 Ordemann J, Jacobi CA, Schwenk W, Stosslein R, Muller JM: Cellular and humoral inflammatory response after laparoscopic and conventional colorectal resections. Surg Endosc 2001;15:600-608.

27 Cardinale F, Chinellato I, Caimmi S, Peroni DG, Franceschini F, Miraglia DG, et al: Perioperative period: immunological modifications. Int J Immunopathol Pharmacol 2011; 24:S3-S12

28 Dhabhar FS, Miller AH, McEwen BS, Spencer RL: Stress-induced changes in blood leukocyte distribution: role of adrenal steroid hormones. J Immunol 1996;157:1638-1644.

29 Elenkov IJ, Wilder RL, Chrousos GP, Vizi ES: The sympathetic nerve - an integrative in terface between two supersystems: the brain and the immune system. Pharmacol Rev 2000;52:595-638.

30 Gan X, Zhang L, Solomon GF, Bonavida B: Mechanism of norepinephrine-mediated inhibition of human NK cytotoxic functions: inhibition of cytokine secretion, target binding, and programming for cytotoxicity. Brain Behav Immun 2002;16:227-246.

-31 Bartal I, Melamed R, Greenfeld K, Atzil S, Glasner A, Domankevich V, et al: Immune perturbations in patients along the perioperative period: alterations in cell surface markers and leukocyte subtypes before and after surgery. Brain Behav Immun 2010;24: 376-386.

32 Hogan BV, Peter MB, Shenoy HG, Horgan K, Hughes TA: Surgery induced immunosuppression. Surgeon 2011;9:38-43.

33 Leaver HA, Craig SR, Yap PL, Walker WS: Lymphocyte responses following open and minimally invasive thoracic surgery. Eur J Clin Invest 2000;30:230-238.

-34 Ogawa K, Hirai M, Katsube T, Murayama M, Hamaguchi K, Shimakawa T, et al: Suppression of cellular immunity by surgical stress. Surgery 2000;127:329-336.
35 Handy JM, Scott AJ, Cross AM, Sinha P, O'Dea KP, Takata M: HLA-DR expression and differential trafficking of monocyte subsets following low to intermediate risk surgery. Anaesthesia 2010;65:27-35.

>36 Evans C, Galustian C, Kumar D, Hagger R, Melville DM, Bodman-Smith M, et al: Impact of surgery on immunologic function: comparison between minimally invasive techniques and conventional laparotomy for surgical resection of colorectal tumors. Am J Surg 2009;197:238-245.

37 Hershman MJ, Cheadle WG, Wellhausen SR, Davidson PF, Polk HC Jr: Monocyte HLADR antigen expression characterizes clinical outcome in the trauma patient. Br J Surg 1990;77:204-207.

38 Caille V, Chiche JD, Nciri N, Berton C, Gibot $\mathrm{S}$, Boval B, et al: Histocompatibility leukocyte antigen-D related expression is specifically altered and predicts mortality in septic shock but not in other causes of shock. Shock 2004;22:521-526.

39 Cavaillon JM, Adib-Conquy M: Determining the degree of immunodysregulation in sepsis. Contrib Nephrol 2007;156:101-111.

40 Oczenski W, Krenn H, Jilch R, Watzka H, Waldenberger F, Koller U, et al: HLA-DR as a marker for increased risk for systemic inflammation and septic complications after cardiac surgery. Intensive Care Med 2003;29: 1253-1257.

41 Moselli NM, Baricocchi E, Ribero D, Sottile A, Suita L, Debernardi F: Intraoperative epidural analgesia prevents the early proinflammatory response to surgical trauma: results from a prospective randomized clinical trial of intraoperative epidural versus general analgesia. Ann Surg Oncol 2011;18: 2722-2731

42 Vogelgesang A, Grunwald U, Langner S, Jack R, Broker BM, Kessler C, et al: Analysis of lymphocyte subsets in patients with stroke and their influence on infection after stroke. Stroke 2008;39:237-241.

43 Vermeij FH, Scholte op Reimer WJ, de Man $\mathrm{P}$, van Oostenbrugge RJ, Franke CL, de Jong $\mathrm{G}$, et al: Stroke-associated infection is an independent risk factor for poor outcome after acute ischemic stroke: data from the Netherlands Stroke Survey. Cerebrovasc Dis 2009; 27:465-471

44 Iadecola C, Anrather J: The immunology of stroke: from mechanisms to translation. Nat Med 2011;17:796-808.

-45 Katzan IL, Cebul RD, Husak SH, Dawson NV, Baker DW: The effect of pneumonia on mortality among patients hospitalized for acute stroke. Neurology 2003;60:620-625.

$\checkmark 46$ Haeusler KG, Schmidt WU, Fohring F, Meisel C, Helms T, Jungehulsing GJ, et al: Cellular immunodepression preceding infectious complications after acute ischemic stroke in humans. Cerebrovasc Dis 2008;25:50-58. 